\title{
Terahertz Heterodyne Imaging Part II: Instruments
}

\author{
Peter H. Siegel ${ }^{1,2}$ and Robert J. Dengler ${ }^{2}$ \\ ${ }^{1}$ Beckman Institute, Division of Biology, California Institute of Technology, \\ 1200 E. California Blvd., Pasadena, CA 91125 \\ ${ }^{2}$ Submillimeter Wave Advanced Technology, Jet Propulsion Laboratory, \\ 4800 Oak Grove Drive, Pasadena, CA 91109 \\ phs@caltech.edu
}

\begin{abstract}
$\underline{\text { Abstract }}$
This paper is Part II of a series of articles on Terahertz heterodyne imaging. In Part I introductory information and techniques were presented. In this paper the authors describe four different types of heterodyne imaging instruments that have been established at their facilities. In Part III (to appear in a future issue of this journal) selected applications of these instruments are discussed.
\end{abstract}

\section{Introduction}

Heterodyne imaging techniques were reviewed in Part I of this paper. In this section the authors describe several instruments that have been set up to perform imaging in the wavelength range between $200 \mathrm{GHz}$ and $2.5 \mathrm{THz}$. These instruments are representative of the variety of heterodyne techniques available to the general research community. One of the instruments is based around a commercially available millimeter wave network analyzer. The others can be assembled from components that are available in one or more RF specialty companies [1]. The suite of instruments described is by no means meant to be all inclusive, nor are the instruments inexpensive or easily assembled. However as interest in CW terahertz techniques grows, it is hoped that more vendors will enter the component market and instruments such as ours will become more available. There is still a wide range of applications that have yet to be fully explored with terahertz heterodyne techniques (see Part III of this series) and the instrument complexity and capability is still extremely primitive in comparison with either infrared or even millimeter-wave systems. So far all realized systems take the form of scanned single pixel or modest $(<10)$ multipixel arrays. The submillimeter-wave camera is still one of the author's long sort after dreams [2], but slow progress is being made and perhaps it is not too far fetched to accept the goal of one company representative the author recently communicated with, who envisioned a Terahertz camera on the shelves of K-Mart ${ }^{\circledR}$ within the next ten years! 


\section{Heterodyne Imaging Systems}

For purposes of this discussion active imaging is taken to mean artificial illumination of the scene by an external coherent RF source. Passive imaging will refer to scenes which are illuminated only by the incoherent background. Note that artificially generated incoherent illumination can be added to a scene via background thermal or noise sources. These might include incandescent or heat lamps as well as plasma discharge sources or fluorescent lighting. In either instance, imaging can be via reflection, specular or scatter, or transmission or a combination of both. Heterodyne techniques can be useful for both passive or active imaging, and in fact, in many instances the NE $\Delta \mathrm{T}$ is low enough so as to simultaneously employ the same system for both observing modes by simply turning off (or chopping) the coherent illumination source. Some practical features of heterodyne imaging systems, that may sway the user in a particular application, are as follows. In active imaging using a coherent source, the detection and illumination source bandwidths can be optimized for noise, dynamic range, $\mathrm{S} / \mathrm{N}$ or specific scene attributes (spectral line width for example). From the hardware point of view, since heterodyne instruments already require modest levels (microwatts-to-milliwatts) of source power to serve as the local oscillator, there is little added complexity in duplicating such sources to serve as coherent illuminators at slightly different RF frequencies. Magnitude and phase information are supplied at a frequency which is readily manipulated and above the $1 / f$ knee of electronic components. In the terahertz regime a wide range of planar, dielectric, horn or lens antennas are available for beam forming, and heterodyne rectifying devices can be both impedance and field matched with high efficiency to these antennas. Signal processing is extremely flexible and, although it has not been tried on a large scale as yet, it is possible to form focal plane array cross-correlation receivers that enable interferometric, multipixel subarray beam forming, electronic beam steering or telescope error correction capabilities $[3,4]$. Finally, with the addition of a swept LO, or a swept illumination source and wide IF bandwidth, active heterodyning provides the same wealth of capabilities available in sophisticated radar systems, including range finding, 3D terrain mapping, synthetic aperture synthesis, etc.

Despite significant functional advantages, the difficulties associated with establishing and operating heterodyne instruments in the terahertz regime are substantial. This is due largely to two things. First, a lack of commercial component technology for the RF source and downconverter modules (transmit/receive systems). Although this situation is slowly changing, there are still no (or at least very few) commercial RF parts that can be procured above $1 \mathrm{THz}$. Second, there is no demonstrated technique for forming large multipixel heterodyne imagers at any wavelength above $100 \mathrm{GHz}$. This is due to the complexity of having to deal with three widely different signals within the same detector - LO, RF and IF. The few small ( $\leq 10$ element) heterodyne arrays that have been realized in the submil- 
limeter rely on close packing individual single pixel waveguide receivers with non optimal fill-factors $[5,6,7,8]$. Several clever millimeter wave array systems have been constructed in W-band (generally centered around the $94 \mathrm{GHz}$ atmospheric window) but all are fairly bulky and expensive $[9,10,11,12,13]$. Focal plane direct detection arrays are much easier to realize in this frequency range for the reasons stated, (no LO and simple DC output) and there are many viable designs from the late 1980's to the mid 1990's for submillimeter-wave array concepts developed largely for the radio astronomy community $[14,15,16,17,18,19$,$] . Only a few of these however [20] were ever tested in het-$ erodyne mode. Much less funding, and hence less research activity, has gone into this area (at least in the US) in recent times, in spite of sporadic recurring interest from both defense and civil agencies ${ }^{1}$.

As an introduction to heterodyne imaging instrumentation we describe four systems that have been set up in the author's laboratories - one passive and three active. These are representative of what is currently available in the wavelength range between $1 \mathrm{~mm}$ and 100 microns and serve to illustrate both system tradeoffs and operational pitfalls. They include custom, commercial and mixed custom/commercial implementation. In parallel with these early trials other submillimeter-wave heterodyne imaging systems have been demonstrated $[21,22,23]$ and there are, as mentioned, a variety of lower frequency passive systems that have been deployed for several, including some pioneering work above $100 \mathrm{GHz}$ by JPL's Bill Wilson way back in 1986 [24].

\section{II.1 Active Heterodyne Imager using Fixed RF and LO Sources for $2.5 \mathrm{THz}$}

Our interest in heterodyne imaging began several years ago after completion of an Earth sounding instrument that measures the thermal emission signatures from dozens of upper atmosphere molecules involved in ozone depletion, global water distribution, pollution monitoring and volcanism [25]. That instrument, Aura Microwave Limb Sounder, carries fixed frequency, broad IF, heterodyne radiometers centered near 110, 180, 240, 640 and $2520 \mathrm{GHz}$. In order to explore the imaging capabilities and pitfalls in the higher end of this frequency range we utilized spare parts from this instrument $[26,27]$ and two commercial far-IR gas lasers [28], and assembled an active heterodyne system centered at $2522 \mathrm{GHz}$ $[29,30]$.

\footnotetext{
${ }^{1}$ NASA has been interested in submillimeter-wave heterodyne array technology for many years, driven by receiver requirements for past and future space observatory instruments such as Large Deployable Reflector and more recently Single Aperture Far InfraRed observatory (SAFIR). However, funding in the past five years has gone mostly into high sensitivity sub-4K direct detection arrays. The US Defense Advanced Research Projects Agency (DARPA) has also had at least two recent proposal calls for submillimeter-wave array technology development in support of imaging for security and terrain mapping applications.
} 
LOCAL OSCILLATOR

difluoromethane $\mathrm{CH}_{2} \mathrm{~F}_{2}$
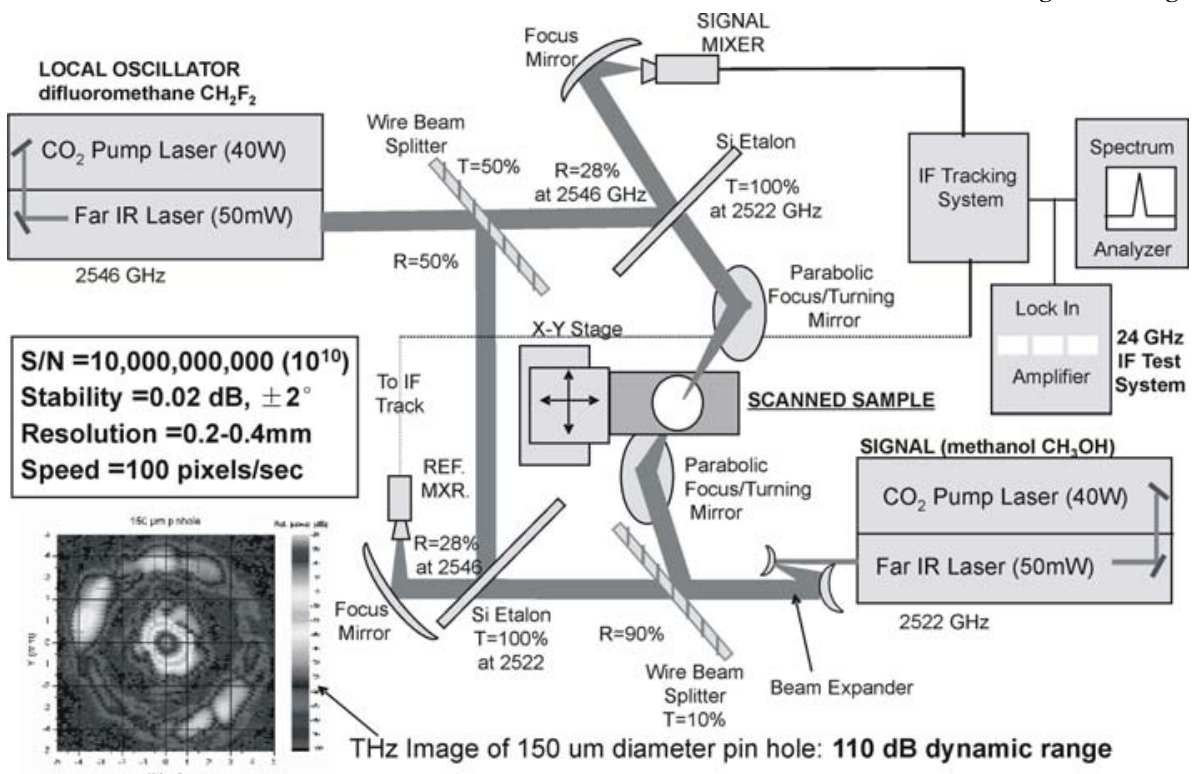

rabolic

Fig. 2 (a). Block diagram of $2.5 \mathrm{THz}$ heterodyne imaging system based on $\mathrm{CO}_{2}$ pumped far IR gas lasers and room temperature Schottky barrier diode mixers.

$\begin{array}{cl}\text { From } 2.5 \mathrm{THz} \text { reference mixer } & \text { IF Portion of Frequency Tracking } \\ -80 \mathrm{dBm} @ 23.713 \mathrm{GHz} & \text { Heterodyne Imager (IF }=24 \mathrm{GHz})\end{array}$
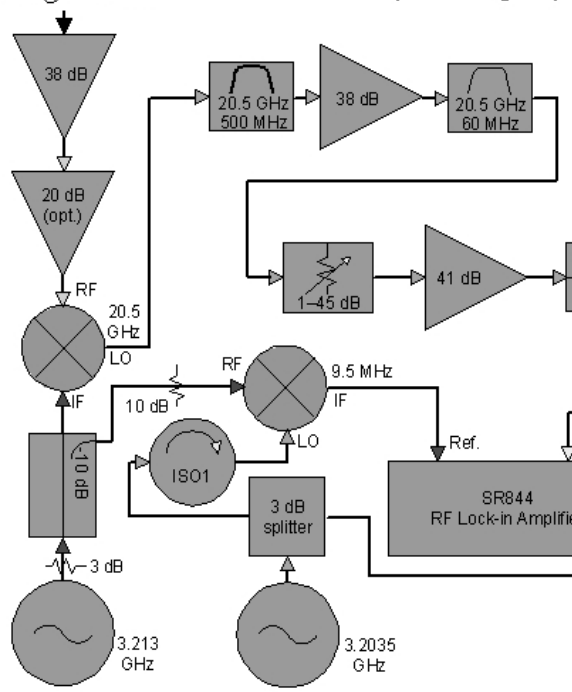

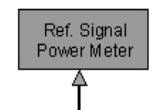

From $2.5 \mathrm{THz}$ signal mixer -30 to $-130 \mathrm{dBm} @ 23.713 \mathrm{GHz}$

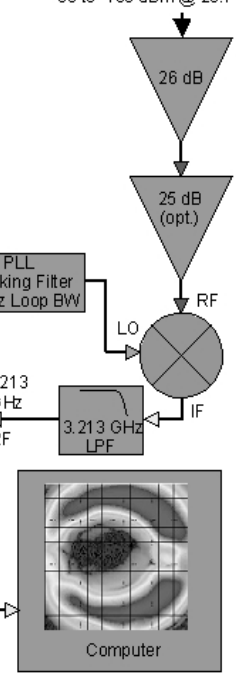

Fig. 2(b). IF portion of heterodyne imaging system showing locking and comparator as well as lock-in detection scheme. 
A block diagram of the imaging instrument appears in Fig. 2a. Key components include the signal and local oscillator sources, consisting of two commercial farIR gas lasers, a far-IR beam expander for producing the desired $f$-number signal beam from the laser output, silicon etalons for RF/LO beam diplexing, two $\mathrm{THz}$ heterodyne down converters (room temperature Schottky diode mixers), two low noise wide band output (IF) amplifiers, a custom microwave phase and amplitude tracking circuit, and a commercial RF lock-in amplifier for recording the detected signal. The imager operates by focusing and then passing the signal at $2522 \mathrm{GHz}$ through a provided sample, and then raster scanning the sample itself (via an X-Y stage) through the fixed beam. After passing through the sample, the output power is collected by a mirror, collimated and then refocused into the Schottky diode mixer, whose IF output power and phase are recorded as a function of sample position. The instrument has a realized dynamic range of $110 \mathrm{~dB}$, a sample acquisition time (limited by the speed of the X-Y positioner) of approximately 100 pixels/second and a focal spot diameter (set by the optical $f$-number and wavelength) of 250 microns. It is worth describing some of the components and subcircuits in more detail as in so doing, some of the advantages and pitfalls of imaging with these techniques will be apparent.

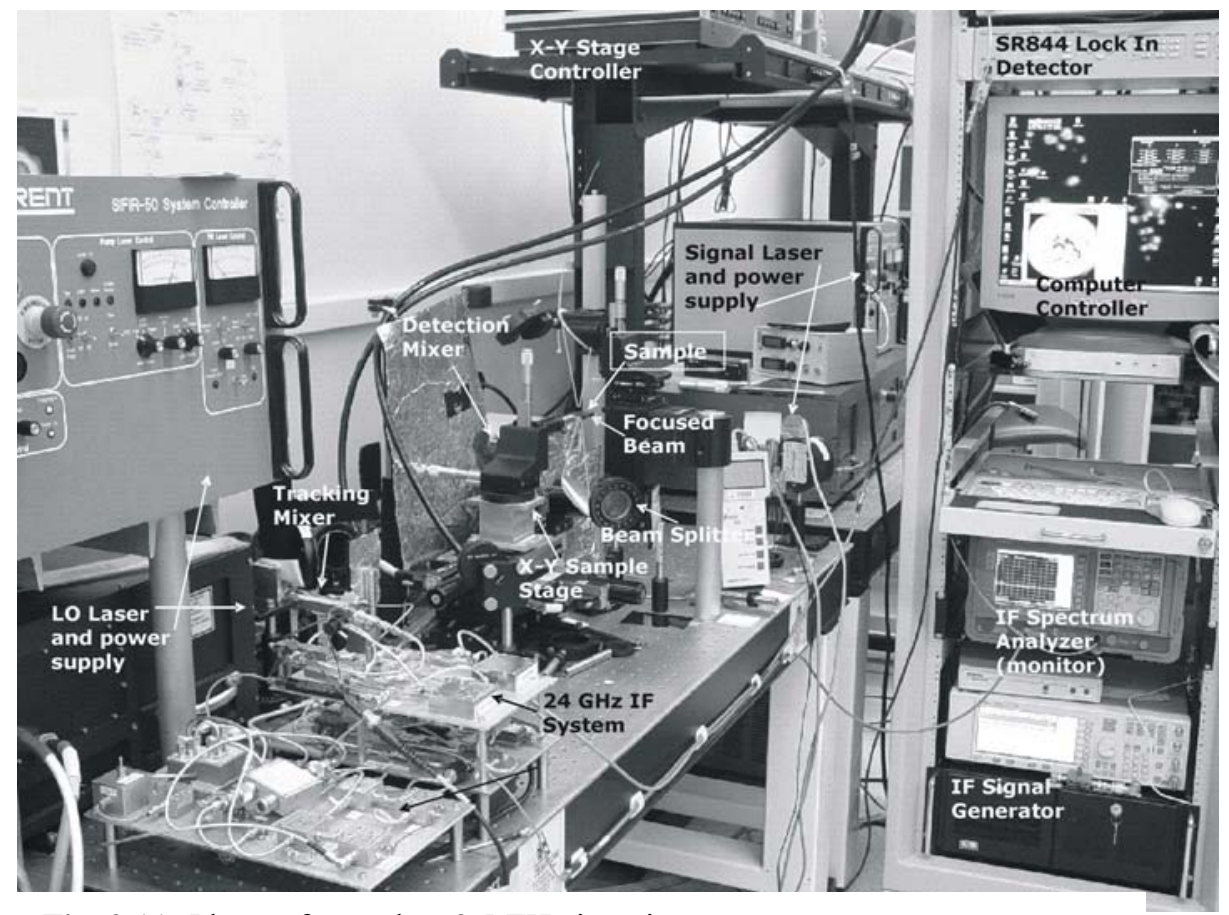

Fig. 2 (c). Photo of complete $2.5 \mathrm{THz}$ imaging system. 
We start with the RF front-end (Fig. 2a). The signal and LO consist of fixed frequency $\mathrm{CO}_{2}$ pumped far-IR gas lasers, one operated on a very strong $(>50 \mathrm{~mW})$ methanol $\left(\mathrm{CH}_{3} \mathrm{OH}\right)$ emission line at $118.83 \mu \mathrm{m}(2522 \mathrm{GHz})$ serving as the signal (lower right), and the other operated on an almost equally strong difluoromethane $\left(\mathrm{CH}_{2} \mathrm{~F}_{2}\right)$ line at $2546 \mathrm{GHz}$, serving as the local oscillator source (upper left) for the heterodyne mixer. The signal laser has an output beam diameter of approximately $5 \mathrm{~mm}$. This is beam expanded to $50 \mathrm{~mm}$ using a custom $\mathrm{z}$-fold arrangement $^{2}$, bounced off a wire grid beam splitter ${ }^{3}$ and focused onto the measurement sample through a commercial off axis parabola ${ }^{4}$. The spot size on the sample is determined by the mirror $f$-number and wavelength as well as the quality of the alignment and focusing, and was measured to be approximately 250 microns (full-width, half-power). After passing through the sample, the expanding beam is collected through a second parabola, collimated and sent through a silicon etalon ${ }^{5}$ [31] after which it is refocused into the mixer. At the same time the local oscillator is passed through a wire grid beam splitter then partially reflected off the silicon etalon where it too is focused into the mixer. The mixer [32] operates in a fundamental mode and consists of a room temperature planar Schottky barrier diode embedded in a single-mode fix-tuned waveguide mount with an instantaneous bandwidth of a few percent. It was developed under an in-house program for application on the NASA Aura spacecraft and operates double-sideband with an equivalent input mixer noise temperature of approximately $6,000 \mathrm{~K}$. The input to the mixer is via a built-in dual-mode Pickett-Potter feed horn [33] which produces a high quality Gaussian beam [26]. The mixer downconverts the signal and LO to an IF of $23.7 \mathrm{GHz}$ where it passes through the first stage amplifier and onto the IF portion of the test system.

If the LO and signal lasers were perfectly stable, the test sample could be scanned through the fixed RF beam and the resulting transmitted amplitude and phase (at the IF) recorded as a function of position using any of a number of microwave detection schemes. However, because both far-IR laser amplitudes drift substantially with time due to changes in gas pressure, slight shifts of the $\mathrm{CO}_{2}$ mode peak, and changes in room humidity, using a single mixer without a separate signal calibration path results in large uncertainties in the transmitted power versus time. To eliminate this problem a phase and amplitude tracking circuit, based on a system originally described by Doane [34], was implemented. On the RF side this involves adding a signal reference path using a second mixer and etalon (as well

\footnotetext{
${ }^{2}$ Electro Optical Components, Inc., 5460 Skylane Blvd., Santa Rosa, CA 95403.

3 Johann Wantaugh, Advanced Spectroscopy Technologies, 12021, Wilshire Blvd., Suite 419, Los Angeles, CA 90025.

4 Melles Griot, 2051 Palomar Airport Road, Carlsbad, California 92011 or Janos Technology Inc., 55 Black Brook Rd., Keene, NH 03431.

${ }^{5}$ The etalons were made from $50 \mathrm{~mm}$ diameter float zone silicon wafers $(>20,000$ ohm-cm) available from Topsil Semiconductor Inc., Frederikssund, Denmark and then lapped to their required thickness (387.5 and 391.1 microns) by Boston Piezo Optics, Inc., Bellingham, MA, USA.
} 
as the two wire grid beam splitters already mentioned). The signal laser is now split into a measurement and reference path using the wire grid and second etalon (lower portion of Fig. 2a). Similarly a portion of the LO laser is picked off and combined with the signal reference in the second mixer. Each mixer now converts to the same IF frequency but the signal beam passes through the sample (adding phase delay and decreasing amplitude) while the reference beam does not. In the IF tracking portion of the imager, the reference IF is mixed with a $3.2 \mathrm{GHz}$ oscillator, filtered, amplified, filtered again by a tracking PLL filter (BW=1 MHz), reamplified and finally applied to the LO input of another mixer whose RF input is the downconverted power from the signal mixer. The output of this mixer is a 2 nd IF signal equal in instantaneous frequency to the $3.2 \mathrm{GHz}$ reference arm oscillator (hence very narrow linewidth) but differing in amplitude and phase depending on the loss and phase shift imposed on the signal beam by the scanned sample. This and the reference oscillator are further downconverted to approximately $9 \mathrm{MHz}$ so they can be inputs to the reference and signal ports of a commercial RF lock-in amplifier (Stanford Research SR844 - peak operating frequency performance below $10 \mathrm{MHz}$ ). In addition to having a maximum two millisecond latency between the issuance of a trigger command to it and the actual logging of data, the lock-in amplifier can also measure the relative phase difference between the reference and measurement IF signals, thereby providing phase delay imagery of the samples. The original tracking circuit described in Doane [34] specified the use of isolators at the RF inputs of the mixers to reduce the amount of signal reflected off of the mixer inputs. Without such isolators a small amount of signal laser power that bypasses the sample plane via the reference mixer path can (and always does!) end up appearing at the measurement mixer input. This can greatly limit the dynamic range of the instrument. Initially, due to this problem (and before we started using the silicon etalons), our dynamic range was only $45 \mathrm{~dB}$. The etalons however have such low reflectivity when their throughput angle is optimized, that we were able to obtain an additional 40 to $50 \mathrm{~dB}$ of isolation between the two THz mixers. A maximum dynamic range of $110 \mathrm{~dB}$ was eventually obtained with the system shown in Fig. 2. Operation at frequencies other than 2.5 $\mathrm{THz}$ is possible by judicious choice of closely spaced (within the mixer IF band) laser lines [35] and replacement of the two Schottky diode mixers with downconverters optimized for these alternate frequencies. Sensitivity enhancement is also possible (at the expense of high-end dynamic range) by replacing the Schottky mixers with more sensitive hot electron bolometer [36] or superconductorinsulator-superconductor mixers [37]. Factors of 10-100 in low-end signal acquisition are possible with these near quantum limited receiver elements. Finally, significant improvement in frequency agility and portability might soon be achieved by replacing the two far IR gas lasers with newly available semiconductor quantum cascade lasers [38,39].

In summary, for applications above $1 \mathrm{THz}$ and up to frequencies as high as these or newer sources and mixers can reach, this implementation for realizing a heterodyne imager will provide very high dynamic range and convenient room temperature operation. Images and applications from this system are given in Part III 
of this paper. However, at some stage this system should be replaced by the $\mathrm{THz}$ equivalent of the unit described next.

\section{II.2 Active Heterodyne Imager Using a Commercial Submillimeter-Wave VNA}

For fixed frequency or very modest bandwidth $(<1 \%)$ swept frequency imaging applications between $200 \mathrm{GHz}$ and $1 \mathrm{THz}$ it is possible to make use of an existing commercial instrument. The vector network analyzer (VNA) has been a primary instrument for RF engineers for more than 40 years [40]. Based around phase locked or directly synthesized frequency transmit/receive ports and heterodyne operation it can measure magnitude and phase with extremely wide dynamic range (up to $100 \mathrm{~dB}$ ), amplitude stability in excess of $0.1 \mathrm{~dB}$ and degree level phase accuracy. Commercial VNA's operating up to $100 \mathrm{GHz}$ have been available for more than a decade from distributors like Agilent $^{6}$ (formerly Hewlett Packard) and Anritsu ${ }^{7}$. AB Millimetre ${ }^{8}$ introduced a submillimeter-wave VNA in the 1980's that has been used at frequencies up to and slightly beyond $1 \mathrm{THz}$. This system has compact $\left(20 \times 10 \times 10 \mathrm{~cm}^{3}\right)$ transmit and receive heads that are coupled to the synthesizer and signal control, processing and phase lock systems through flexible coaxial cables that allow easy movement and positioning of the source and detector elements. Signal power is generated from a phase locked oscillator at $\mathrm{W}$-band $(100 \mathrm{GHz})$ which is frequency multiplied in harmonic steps to the desired output using a solid state upconverter. The proper output harmonic must be selected by employing a waveguide high pass filter implemented in waveguide. The receive head employs a harmonic mixer driven by an identical Gunn oscillator (the harmonic mixer downconverts the input RF by heterodyning with the nth harmonic of the oscillator). Again, waveguide high pass filters are required to isolate the proper harmonic for the mixing process. RF signals are transmitted and received via free space using well controlled single mode waveguide horns. The system has high signal-to-noise, a dynamic range of 60-90 $\mathrm{dB}$ and mechanical frequency tuning with limited sweep capability $(\sim 0.5 \%)$. Sweep limitations stem from the use of W-band Gunn diode oscillators ${ }^{9}$ in the signal and source arms which have limited electronic tuning range, but very wideband mechanical tuning $(>30 \%)$. These oscillators are multiplied up in frequency using solid-state Schottky diode multipliers to generate the source power $\left(7^{\text {th }}\right.$ or slightly higher harmonics are possible) and are also used to supply the power for the harmonic mixer used on the receive side. The multiplier and mixer in our unit were fabricated by RPG Radiometer Physics ${ }^{10}$ and require extensive tuning (4 tuning knobs on each component) at every frequency, but these can be replaced by broad band tunerless components from Virginia Diodes ${ }^{11}$. We have also util-

\footnotetext{
${ }^{6}$ Agilent Technologies, 395 Page Mill Rd. Palo Alto, CA 94306.

${ }^{7}$ Anritsu Microwave Measurements Division, 490 Jarvis Dr. Morgan Hill, CA 95037

${ }^{8}$ MVNA ESA-1/ESA 2, AB Millimetre, 52 Rue Lhomond, 75005, Paris, France.

${ }^{9}$ Oscillators obtained from John Carlstrom, U. Chicago, IL, USA

${ }^{10}$ RPG Radiometer Physics, Bergerwiesenstrase 15, Meckenheim 5309, Germany.

${ }^{11}$ Virginia Diodes, 979 Second St., Suite 309, Charlottesville, VA, 22902 USA
} 
ized the MVNA with phase locked backward wave oscillator tubes (BWO) ${ }^{12}$ up to $350 \mathrm{GHz}$ to replace the Gunn and multiplier on the source side, yielding wider swept frequency capability $(30 \mathrm{GHz})$, and in principle it is possible to extend the frequency by following the BWO with a harmonic multiplier. The quality of the phase lock with the backward wave tubes is not as good above $300 \mathrm{GHz}$ however, and of course their mass and size greatly reduces the portability of the transmit/receive units. A second commercial vendor, Oleson Microwave (OML) ${ }^{13}$, has now produced frequency extension heads for the Agilent and Anritsu VNAs that allow the user to reach $330 \mathrm{GHz}$ with full waveguide band sweep capability $(30 \%)$ and this company plans to introduce units to $500 \mathrm{GHz}$ within the next year.

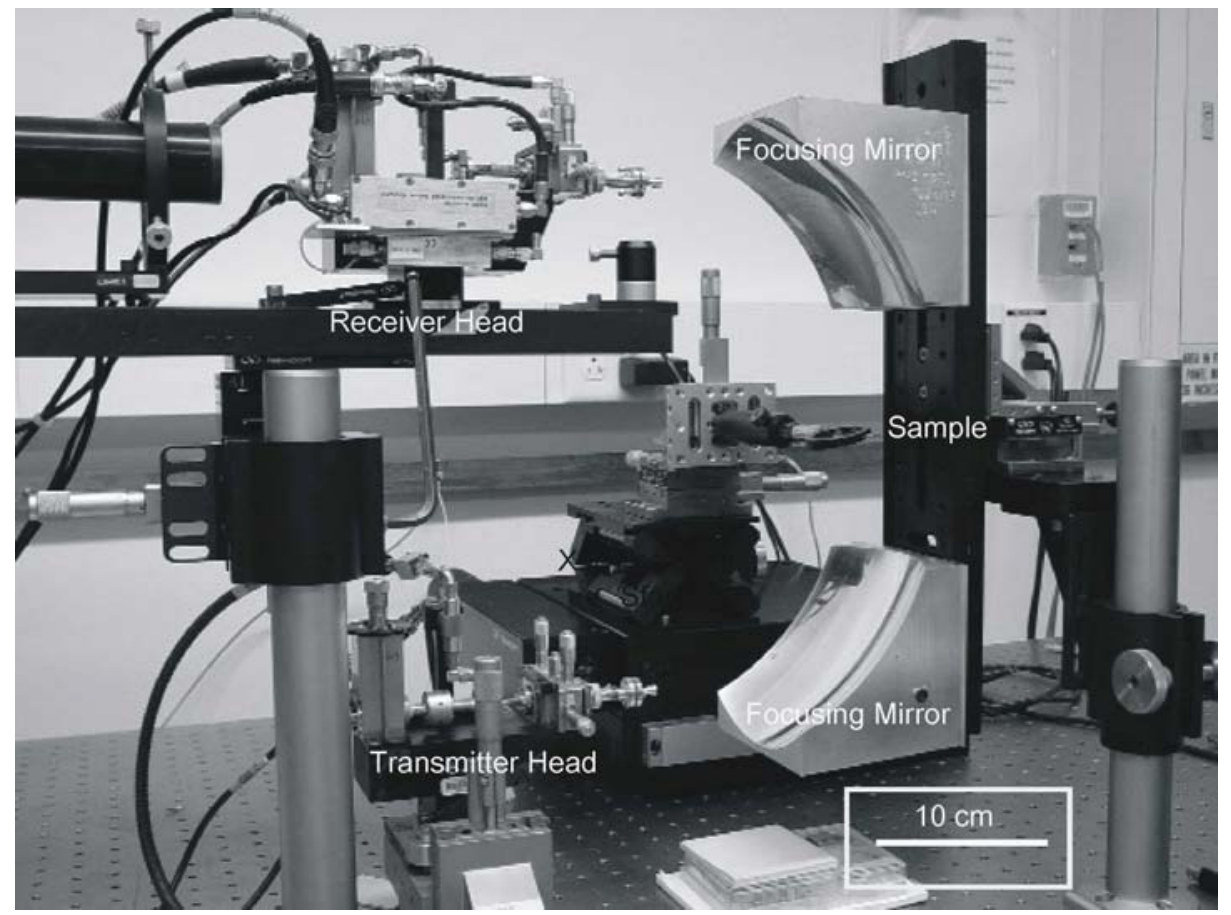

Fig. 3(a). Photo of transmit/receive heads, focusing elements and sample stage of MVNA based imaging system.

Using a few simple modifications and some custom developed imaging and display software (available from the authors) we have converted the AB Millimetre MVNA into a frequency flexible submillimeter wave imaging system. The conversion was accomplished by adding a second image acquisition computer operating in concert with the supplied MVNA control computer. This computer controls an X-Y stage that scans the sample through a fixed beam and collects signal amplitude and phase information directly from analog output ports on the MVNA.

\footnotetext{
${ }^{12}$ Insight Products, PO Box 297, Brighton, MA 02135, USA

${ }^{13}$ OML, Inc., 300 Digital Dr. Morgan Hill, CA 95037.
} 


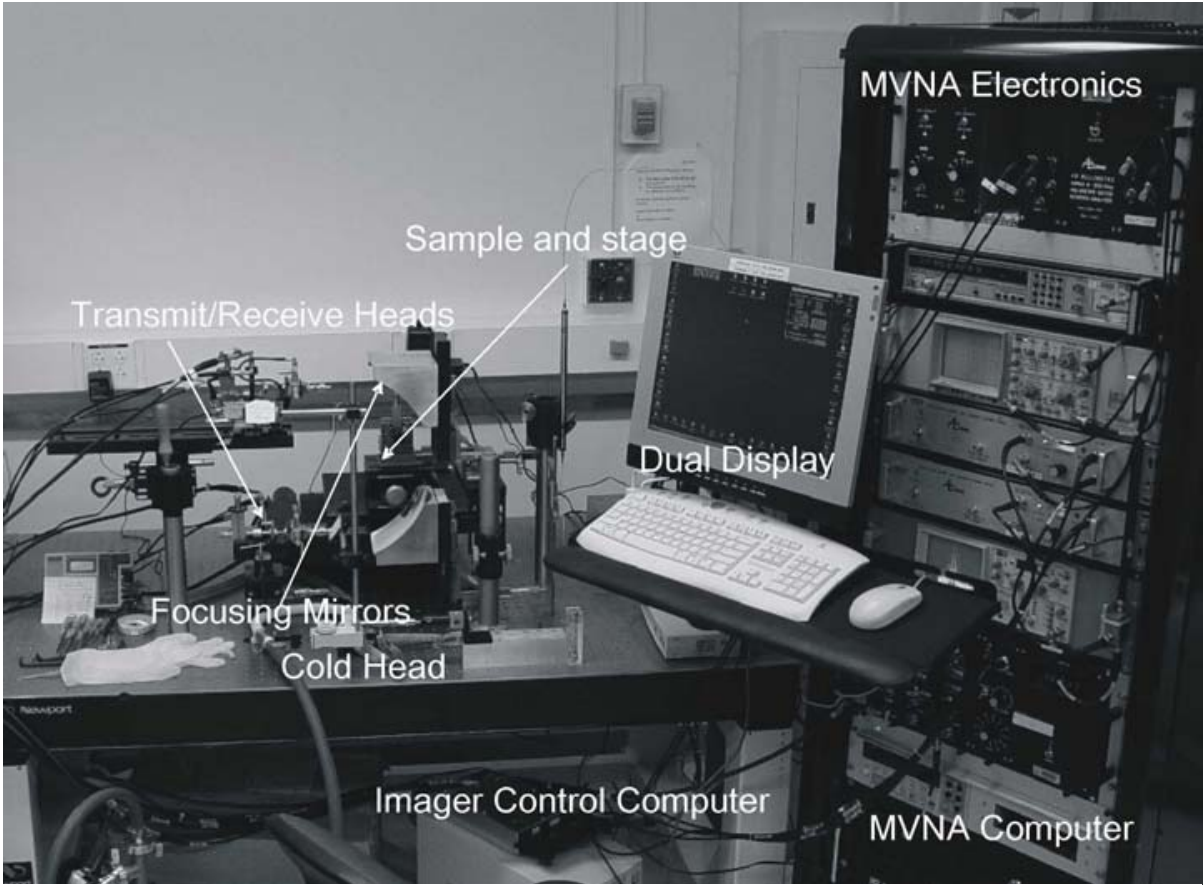

Fig. 3(b). Photo of complete MVNA based imager with control rack.

The image data is displayed on the acquisition computer in real time as it is collected. Post acquisition software is then used to normalize the data to either the maximum signal found in the image, or to a reference position, and correct for signal position errors caused by the effective time constant of the MVNA. All other functions are controlled by the MVNA including frequency selection and locking, power peaking, signal integration and averaging. The MVNA control computer and image acquisition computer run simultaneously but use a single monitor controlled via a simple keyboard-video-mouse (KVM) switch. Since the stability of the MVNA and the source/sampling units are phase locked to a common synthesizer there is no need to add a reference path in order to achieve high stability as in the case with the gas laser based imager. However, absolute phase information, or phase referred to a reference path is not available unless such a modification is implemented.

The assembled instrument can acquire a maximum of about 40 pixels per second based on trade-offs between noise level and signal averaging time. Our unit has an operating range of $100-700 \mathrm{GHz}$ but newer models extend the upper limit to near $1 \mathrm{THz}$. Amplitude stability is typically $0.2 \mathrm{~dB}$ but can be increased with longer data averaging. Phase resolution was found to be about 4 degrees and a dynamic range of $70 \mathrm{~dB}$ was typically achieved up to $550 \mathrm{GHz}$ and $60 \mathrm{~dB}$ up to $750 \mathrm{GHz}$. Again, improved performance is advertised for newer models. The imaging MVNA system has been used for both reflection and transmission measurements using the setups displayed in the pictures in Fig. 3. Images and applica- 
tions are given in Part III of the paper. This commercial MVNA system is an ideal way of implementing a heterodyne imager with enormous frequency range and hopefully in the near future with frequency sweep capability as well.

\section{II.3 Active Heterodyne Imager with Swept Frequency Signal and LO Sources}

One of the major advantages of heterodyning is the capability to measure very small frequency changes in an incoming signal. The ultimate resolution is directly tied to the frequency stability of the LO which in most cases can be phase locked to an ultra stable reference operating in the $\mathrm{MHz}$ region. High quality quartz oscillators have short term stability of 1 part in $10^{12}$ at $10 \mathrm{MHz}$, which yields a frequency resolution of one part in $10^{7}$, i.e. $100 \mathrm{kHz}$, at $1 \mathrm{THz}$, with higher values possible. For resolving and measuring the linewidth of a natural or artificial signal source, this spectral frequency resolution, $\mathrm{R} \equiv f_{c} / \mathrm{B}_{\text {out, }}$ where $\mathrm{B}_{\text {out }}$ is the detection bandwidth (generally arranged to be much smaller than $\mathrm{B}_{\mathrm{IF}}$ ), is very hard to match with any other technique. We have used the instrument described in IV.1 to make the first sub-MHz linewidth measurements of quantum cascade lasers [41] operating near 3.5 THz for example. The narrow frequency resolution also allows one to passively resolve the Doppler velocity $(\mathrm{v}=\mathrm{c} / 2 \mathrm{R})$ of objects moving towards or away from the receiver to within a few meters per second $(15 \mathrm{~m} / \mathrm{sec}$ in this example at $1 \mathrm{THz}$ or $\sim 54 \mathrm{~km} / \mathrm{hr}$ ). The same components provide very high phase resolution as well. With our fixed frequency $2.5 \mathrm{THz}$ system, in a reasonably stable environment, we have found that it is possible to measure the phase change of the signal upon transmission through an object with a relative accuracy of approximately 1 part in 50 or $2 \%$. Therefore if the object is changing position in the scene relative to the transmitter or receiver, the shift in position can be measured in reflection with an accuracy of about $1 / 50^{\text {th }}$ of a wavelength or 1 micron at $2.5 \mathrm{THz}$ ( 2 microns round trip). However any turbulence or instability in the path between the transmitter and receiver will dramatically degrade this performance. Also there is no simple way of referencing an observed phase shift to an absolute or even relative zero without a length variable reference path, since phase shifts greater than $\pi$ radians simply repeat in the receiver. However if the signal is swept over a modest bandwidth it is possible to measure the progressive phase change that occurs as the wavelength varies. The phase change with frequency, $\Delta \Phi / \Delta f_{\mathrm{RF}}$, is directly translatable into a measurement of the index of refraction, $n$, in the case of transmission through an object:

$n=\left(\frac{\Delta \Phi}{\Delta f_{R F}} \cdot \frac{c}{360 \cdot l}+1\right)$ where $l$ is the thickness and $\Delta \theta / \Delta f_{\mathrm{RF}}$ can be determined

from a straight line fit to the phase versus frequency data. The swept system can also be used in ranging, since a change in RF signal frequency translates into a change in the phase of the signal reflected off an object at a distance $l$ :

$\frac{\Delta \Phi}{\Delta f_{R F}}=\frac{360 \cdot 2 l}{c}$, where for example, if $\Delta \Phi$ is 180 degrees and $l$ is $1 \mathrm{~km}$, the change in frequency that must be resolved is $75 \mathrm{kHz}(75 \mathrm{MHz}$ at $l=1 \mathrm{~m})$, well within the range of a $\mathrm{CW} \mathrm{THz} \mathrm{system.}$ 
In order to examine the potential applications for a swept frequency RF heterodyne system we constructed the system shown in Fig. 4(a). Like the MVNA it uses multiplied submillimeter-wave sources derived from low frequency synthesizers. The phase reference is generated at microwave frequencies, reducing the amount of submillimeter-wave hardware required. By judicious choice of IF frequencies, a significant amount of inexpensive commercial communications hardware can be utilized in the instrument. The system employs an electronically tuned and locked source and LO to produce a fixed IF as the RF frequency is swept. An alternative arrangement would be to use a very wide IF with a tunable narrowband filter, a fixed LO and a swept signal.

Looking at Fig. 4 (a), the RF signal is generated by a X36 frequency multiplier

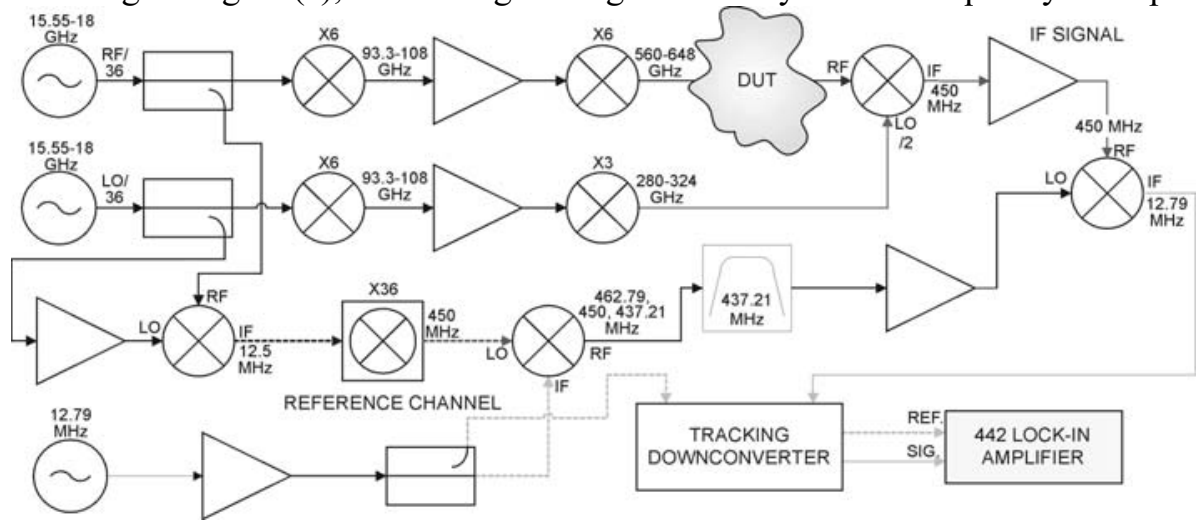

Fig. 4 (a). Block diagram of active swept $600 \mathrm{GHz}$ imaging system.

sequence in order to realize the electronic tuning with a commercial microwave synthesizer. A $640 \mathrm{GHz}$ subharmonically-pumped mixer [42] is used for the signal downconversion. The mixer is actually operated in a single-diode $2^{\text {nd }}$ harmonic mode $\left(f_{\mathrm{IF}}=f_{\mathrm{RF}} 2 f_{\mathrm{LO}}\right)$ by biasing one of the two diodes to the on state, rather than the designed anti-parallel-pair subharmonic mixing mode, to achieve greater $\mathrm{RF}$ bandwidth at the expense of noise performance [43]. The signal, as well as the LO for the mixer are derived from a pair of 14-18 GHz synthesizer modules having a step size of $250 \mathrm{kHz}$. Both synthesizers are multiplied up to the millimeter band (84-108 GHz) using Millitech ${ }^{14}$ X6 active multipliers. The resulting Wband signals are then amplified by $20 \mathrm{~dB}$ using a pair of MMIC power amplifiers [44,45] developed for the ESA Herschel Space Telescope HIFI instrument [46]. The amplified RF is then multiplied again by X6 (signal), or X3 (LO), by a set of custom frequency doublers and triplers also developed for HIFI [47]. The LO and signal frequencies are synchronized so as to generate a constant IF frequency of $450 \mathrm{MHz}$ at the harmonic mixer IF output. Both signal and LO synthesizers are controlled from a Windows PC via a 4 wire synchronous serial interface, using the PC parallel port and a custom driver written in Visual Basic.

${ }^{14}$ Millitech, Inc., 29 Industrial Drive East, Northampton, MA 01060. 

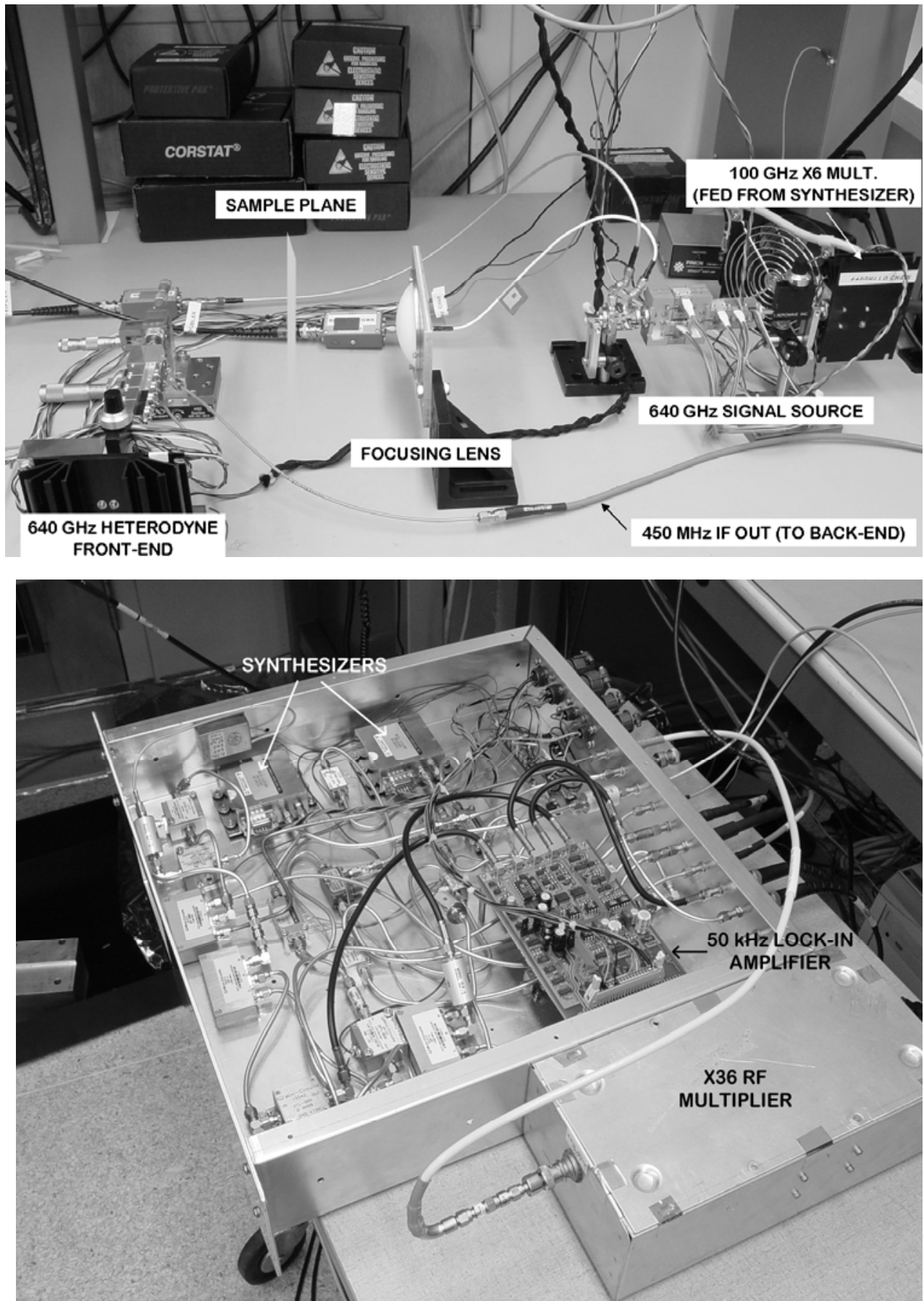

Fig. 4(b). TOP: Photo of compact all-solid-state swept frequency $600 \mathrm{GHz}$ imager showing heterodyne receiver, local oscillator and signal driver sources and a focusing lens for image acquisition. All components are hand-held and positionable. BOTTOM: Control box and complete electronics module (minus display). 
Looking at Fig. 4 (a), the RF signal is generated by a X36 frequency multiplier sequence in order to realize the electronic tuning with a commercial microwave synthesizer. A $640 \mathrm{GHz}$ subharmonically-pumped mixer [48] is used for the signal downconversion. The mixer is actually operated in a single-diode $2^{\text {nd }}$ harmonic mode $\left(f_{\mathrm{IF}}=f_{\mathrm{RF}}-2 f_{\mathrm{LO}}\right)$ by biasing one of the two diodes to the on state, rather than the designed anti-parallel-pair subharmonic mixing mode, to achieve greater RF bandwidth at the expense of noise performance [49]. The signal, as well as the LO, for the mixer are derived from a pair of $14-18 \mathrm{GHz}$ synthesizer modules having a step size of $250 \mathrm{kHz}$. Both synthesizers are multiplied up to the millimeter band (84-108 GHz) using Millitech ${ }^{15}$ X6 active multipliers. The resulting Wband signals are then amplified by $20 \mathrm{~dB}$ using a pair of MMIC power amplifiers $[50,51]$ developed for the ESA Herschel Space Telescope mission - Heterodyne Instrument for the Far Infrared (HIFI) [52]. The amplified RF is then multiplied again by X6 (signal), or X3 (LO), by a set of custom frequency doublers and triplers also developed for HIFI [53]. The LO and signal frequencies are synchronized so as to generate a constant IF frequency of $450 \mathrm{MHz}$ at the harmonic mixer IF output. Both signal and LO synthesizers are controlled from a Windows $\mathrm{PC}$ via a 4 wire synchronous serial interface, using the $\mathrm{PC}$ parallel port and a custom driver written in Visual Basic.

The low-cost synthesizers used in this system have poor phase noise characteristics $(-54 \mathrm{dBc} / \mathrm{Hz} @+/-1 \mathrm{kHz})$, and as a result the highly multiplied $450 \mathrm{MHz}$ IF signals are actually indiscernible from the accompanying phase noise when viewed on a spectrum analyzer. Therefore it was necessary to reference both the signal and reference IF's to a stable source before detection [34]. This is accomplished by shifting the $450 \mathrm{MHz}$ reference $12.79 \mathrm{MHz}$ down in frequency using a mixer and bandpass filter, and then mixing the resultant $437.21 \mathrm{MHz}$ signal with the $450 \mathrm{MHz}$ IF signal. Since the phase noise of the shifted $437.21 \mathrm{MHz}$ and 450 $\mathrm{MHz}$ IF signals is totally correlated, mixing the two signals cancels out that noise, leaving a $12.79 \mathrm{MHz}$ signal that has the same amplitude and phase characteristics (minus the synthesizer noise) as the $450 \mathrm{MHz}$ IF signal. It should be noted that any $450 \mathrm{MHz}$ reference signal passing through the $437.21 \mathrm{MHz}$ bandpass filter will be downconverted at the $450 \mathrm{MHz}$ signal mixer, resulting in crosstalk and loss of dynamic range. It is therefore absolutely essential that the $437.21 \mathrm{MHz}$ filter have extremely high rejection at $450 \mathrm{MHz}$. The chosen filter, a Motorola TFE6213A (extracted from a Motorola "Micor" communications radio) is very small yet has over $100 \mathrm{~dB}$ of rejection at $450 \mathrm{MHz}$. The relatively high loss of this filter $(10 \mathrm{~dB}$, due to the small size vs. high $\mathrm{Q})$ is easily compensated for by additional amplification. The $12.79 \mathrm{MHz}$ signal and reference channels are finally converted to $\sim 66 \mathrm{kHz}$ for detection by a Scitec ${ }^{16} 442$ lock-in amplifier using a $12.86 \mathrm{MHz}$ oscillator, isolation amplifiers and couplers, and mixers. The lock-in

\footnotetext{
${ }^{15}$ Millitech, Inc., 29 Industrial Drive East, Northampton, MA 01060.

${ }^{16}$ Scitec Instruments Ltd., Bartles Industrial Estate, North Street Redruth, Cornwall TR15 1HR, United Kingdom.
} 
amplifier functions as a variable bandwidth magnitude and phase receiver. Bandwidth and gain are also controlled by a Windows PC via a 9 bit parallel interface, while the vector X and Y DC outputs of the lock-in amplifier are read by an analog data acquisition board in the $\mathrm{PC}$ and converted to polar format.

At maximum detection bandwidth, real-time acquisition speeds of over 3000 points per second are possible. The instrument has a phase stability of \pm 2.5 degrees over a frequency range of $560-640 \mathrm{GHz}$ and an amplitude stability of $<0.1$ $\mathrm{dB}$. The dynamic range exceeds $60 \mathrm{~dB}$ over the range $560-625 \mathrm{GHz}$ and reached $90 \mathrm{~dB}$ at some points. To test the performance of the instrument and verify accurate phase measurement capability, the relative phase delay of a Teflon slab was measured from 560 to $640 \mathrm{GHz}$ by introducing the slab between the submillimeter-wave source and mixer. A highly accurate determination of the rate of change of phase with respect to frequency, or time delay, was obtained as shown in Fig. 4 (c). From the data $\Delta \Phi / \Delta f=7.618$ degrees $/ \mathrm{GHz}$, yielding a measured dielectric constant of 2.063 . This is within $1 \%$ of the highly accurate measurements of [54]. The stability and dynamic range of the system is shown by the plot in Fig. 4(d).

The swept system can be used for recording broad spectral features, real time index measurements, acquiring and comparing images at more than one frequency (to reduce speckle for example) and for object ranging. The achieved dynamic range of $60 \mathrm{~dB}$ over much of the frequency band is limited by the harmonic mixer performance which was not designed for this application (intended to be narrow band high sensitivity). This problem is expected to be rectified with our next generation broadband subharmonic mixer modules (currently in fabrication). To date no RF images have been taken with the swept system as we are waiting for completion of an optical Fresnel lens for beam forming. It should be pointed out that an additional benefit of a frequency scanned system when using a single pixel to acquire an image, is the possibility of using a dispersive lens designed to spread the transmit/receive beams over the scene in one dimension as a function of frequency. A complete image can then be acquired via frequency sweeping and mechanical scanning in only one dimension. This technique is used in grating spectrometers and very cleverly in the passive millimeter wave imager developed by ThermoTrex [55]. 

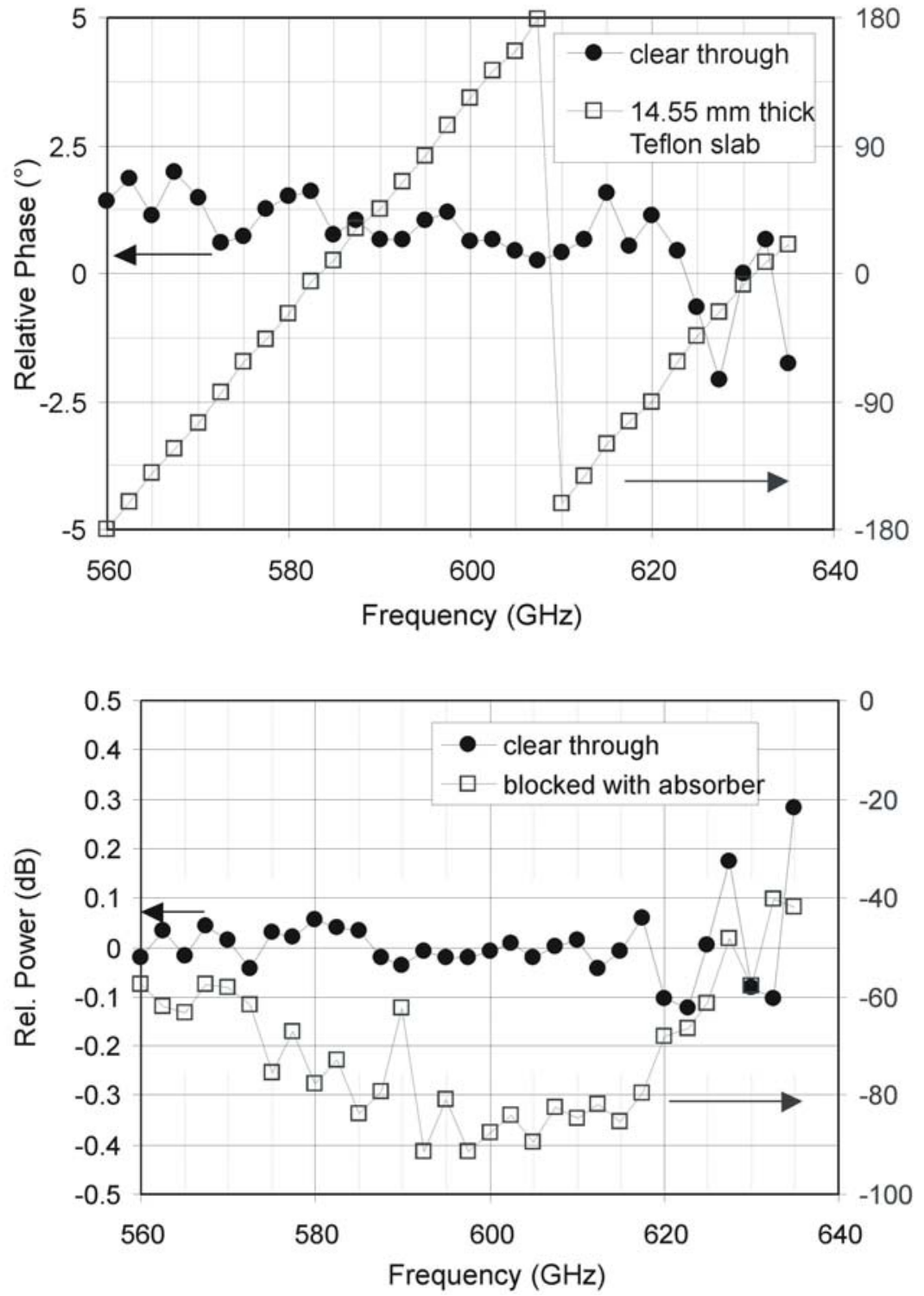

Fig. 4 (c) Top: Phase vs. frequency plot for transmission through a Teflon slab from 560 to $640 \mathrm{GHz}$. (d) Bottom: Noise floor and amplitude stability vs. frequency. 
II.4 Passive Heterodyne Imaging using Radiometers

Heterodyne systems can also be used to advantage in passive imaging where the thermal energy from the scene is captured and analyzed using broadband detection schemes. Contrast comes from varying amounts of reflected energy that emanates from natural sources such as the sun (hot), the sky (cold) or the surrounding terrain. Indoors this includes incandescent or fluorescent light sources. The advantages of passive imaging are that an irradiating source does not have to be utilized, which reduces the risk of detection (in a military application for example), and that personnel and materiel are not exposed to radiation that may be, or at least may be perceived as being, dangerous. There is also no source of speckle with a passive system, diffraction between reflections off different details of an observed object within a single pixel, or specular reflection, in which the directed source and receiver appear at equal angles with respect to the normal to the object, both of which can be major problems in active imaging. To maximize signal power in this mode, the IF bandwidth of the receiver should be as wide as possible $\left(\mathrm{P}_{\text {scene }} \sim k \mathrm{~TB}_{\mathrm{IF}}\right)$. Very broad band $(>150 \mathrm{GHz})$ amplifiers have been demonstrated [56] and at terahertz frequencies these still represent only modest RF fractional bandwidths. The trade-off comes in the available noise temperature of the IF amplifier, $T_{I F}$ which tends to increase with bandwidth and upper operating frequency. This drives the system temperature (not including optical path losses and antenna noise contributions): $\mathrm{T}_{\mathrm{sys}} \approx \mathrm{T}_{\text {mixer }}+\mathrm{LT}_{\mathrm{IF}}$, where $\mathrm{L}$ is conversion loss in the mixer and $\mathrm{T}_{\text {mixer }}$ is the equivalent input noise temperature (a combination of thermal, shot, quantum and scattering noise in the diode or detector element). The minimum detectable temperature, $\Delta \mathrm{T}$, which is more generally written for radiometric systems as $\mathrm{T}_{\mathrm{sys}} /\left(\mathrm{B}_{\mathrm{IF}} \tau\right)^{1 / 2}$, where $\tau$ is the integration time in seconds, improves with the square root of the IF bandwidth, and is the major system driver in the heterodyne system. Since we are now measuring broad band signals in a region of the blackbody curve where ambient temperature sources have very low thermal emission energy, it may be very useful to increase scene brightness using incoherent illumination either from a hot thermal back body source (like a heater lamp) or a strong noise source (like a plasma discharge). The particular frequency chosen for the radiometric system is a complex trade off between system performance, optical resolution, atmospheric absorption and thermal contrast in the scene. A large body of development work has gone on in passive thermal imaging at millimeter wavelengths [57], and has already been mentioned several complete systems have been deployed for over a decade.

Again utilizing spare parts from our recent space-flight programs we assembled the passive imager shown in Fig. 5 operating at a center frequency of $640 \mathrm{GHz}$ [58]. This frequency seems to be a good trade-off between system performance, optical resolution and atmospheric transmission loss. A slightly better atmospheric transmission window exists near $850 \mathrm{GHz}$ but our component hardware did not cover this frequency range. Since no signal source is required, or backend spectrometer (maximized broad band signal only), the instrument is relatively simple and compact. The mixer and LO are similar to those used in the active swept system described previously (section IV.3). A very small (50mm aperture) 
off-axis parabolic mirror (utilized because it was readily available) focuses onto the scene a few meters away, by providing a beam waist at the input horn on the mixer which is slightly in front of the nominal phase center (non paraxial position). The LO center frequency is $642 \mathrm{GHz}$ and the IF amplifier has a $6-18 \mathrm{GHz}$ pass band, yielding conversion bands (upper and lower sidebands) of 624-636 and 648-660 GHz. Since the two converted bands are folded onto each other in the mixing process, the pre-detection bandwidth $\mathrm{B}_{\text {in }}$ is one half of the converted spectrum, or $12 \mathrm{GHz}$. The full IF signal band is fed to a second high-gain IF amplifier and crystal detector, which in turn is read by a lock-in amplifier. By chopping the input signal to the mixer, only the difference in received power between the subject and a fixed RF load is measured. As a result, all drift in the mixer, LO, IF amplifiers and detector is cancelled. The system was calibrated by recording the lock-in amplifier output voltage (corresponding to detected power) with the receiver beam terminated in Eccosorb (blackbody) loads held at $\mathrm{T}_{\mathrm{Hot}}=295$ and $\mathrm{T}_{\text {Cold }}=78 \mathrm{~K}$ respectively. The radiometric noise temperature $\mathrm{T}_{\text {scene }}$ was then calculated in the standard way from:

$\mathrm{T}_{\text {scene }}=\frac{\mathrm{T}_{\text {Hot }}-\mathrm{T}_{\text {Cold }}}{\mathrm{P}_{\text {Hot }}-\mathrm{P}_{\text {Cold }}} \cdot\left(\mathrm{P}_{\text {in }}-\mathrm{P}_{\text {Cold }}\right)+\mathrm{T}_{\text {Cold }}$, where $\mathrm{P}_{\text {Hot }}$ and $\mathrm{P}_{\text {Cold }}$ and $\mathrm{P}_{\text {in }}$ are the

blackbody powers measured by the lock-in amplifier for the hot load, cold load and scene respectively. The image is built up by scanning the antenna and radiometer front end across the scene. A lock-in amplifier integration time of 10 milliseconds was used, giving a maximum acquisition speed of 100 points per second. However, to avoid scan overshoot problems between adjacent pixel rows the scan was slowed down considerably (this problem has been addressed in later versions of the imaging software that was used for the MVNA and active imaging instruments). The RMS noise level, NE $\Delta \mathrm{T}$, for the $10 \mathrm{msec}$ integration time per pixel (plus chopper) was measured to be $0.69 \mathrm{~K}$. This is within a factor of 2 of the prediction from the radiometer equation for a receiver input noise temperature of $3600 \mathrm{~K}$ double-sideband. The difference is due largely to the chopping, which effectively halves the observation time in each reading.

As already mentioned it is possible to enhance the contrast of a passive imager by adding a source of incoherent energy that can be reflected off the objects in the scene. We have tried a simple commercial 150-watt ceramic infrared heater (Lizard Lamp) which has a measured noise temperature very close to its physical temperature (high emissivity). In our case the lamp was not distributed enough across the scene to give strong global illumination, but similar techniques using distributed sources surrounding the subject have been reported with good success [59]. Images made with our $640 \mathrm{GHz}$ system on both a human subject and metallic objects are shown and described in Part III of this paper. 

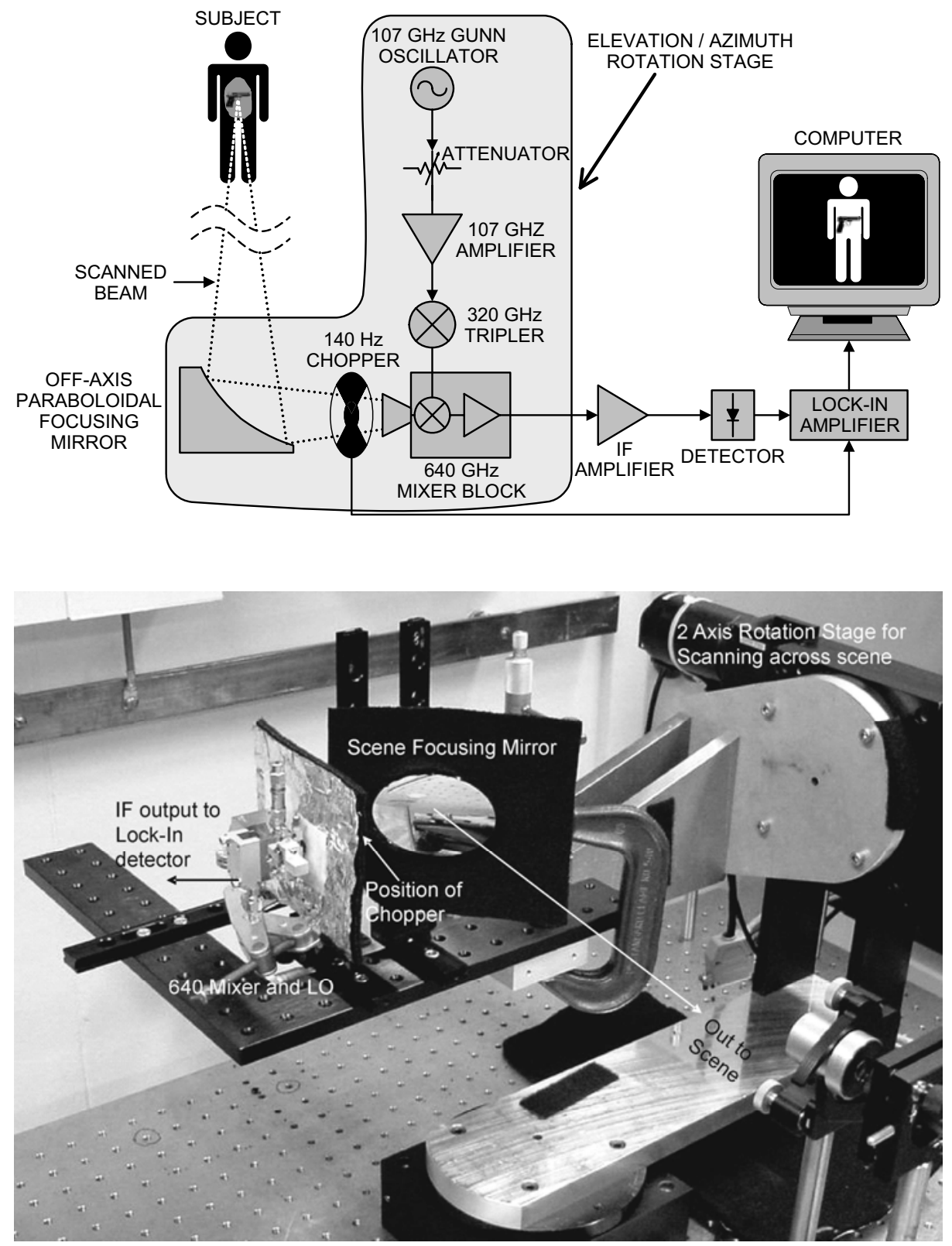

Fig. 5 (a) Top: Block diagram of passive imager. (b). Bottom: Photo of imager front end module. 


\section{Summary}

The authors have described four heterodyne imaging instruments that have been assembled from components and instruments originally developed for space science applications. None of these instruments have the sophistication of the millimeter wave imaging arrays developed for radar and through-the-fog aircraft landing systems, nor the throughput available from widespread commercial infrared camera systems. The potential for heterodyne applications in spectroscopy, radar, security scanning and communication are tantalizing however and it is a good time to begin to take a close look at the enabling component technology that can ultimately lead to more compact, inexpensive, and sophisticated instruments. There is still a great deal of basic phenomenology that must also be investigated in order to understand if the perceived instruments can really deliver the required level of product. In the next part of this series we look at some of the applications that are driving the instrument work in our own laboratories.

\section{Acknowledgements}

The authors would like to thank again all those who they acknowledged in Part I of this paper. As before, this work was carried out at the California Institute of Technology Jet Propulsion Laboratory under contract from the National Aeronautics and Space Administration with additional funding from the National Institute of Health under grant K25 EB00109-01 to the California Institute of Technology Division of Biology.

\section{$\underline{\text { References }}$}

[1]. There are several commercial RF companies that sell components in the submillimeter wave regime. These include (but are not limited to): Agilent Technologies, 395 Page Mill Rd. Palo Alto, CA, 94306 USA; Anritsu Microwave Measurements Division, 490 Jarvis Dr. Morgan Hill, CA 95037 USA; AB Millimetre, 52 Rue Lhomond, 75005 Paris, France; John Carlstrom, U. Chicago, IL, USA; RPG Radiometer Physics, Bergerwiesenstrase 15, Meckenheim 5309, Germany; Virginia Diodes, 979 Second St., Suite 309, Charlottesville, VA, 22902 USA; Insight Products, PO Box 297, Brighton, MA, 02135 USA; OML, Inc., 300 Digital Dr. Morgan Hill, CA 95037 USA; Pacific Millimeter, 64 Lookout Mt. Circle, Golden, CO, 80401, USA; Custom Microwave, 940 Boston Ave., Longmont, CO, 80501, USA; Quinstar, 24085 Garnier St., Torrance, CA 90505 U.S.A.; Farran Technology Ltd., Ballincollig, Cork, Ireland; QMC Instruments Ltd., School of Physics and Astronomy, Cardiff University, 5, The Parade, Cardiff, CF24 3YB, U.K.; ELVA-1, Millimeter Wave Div., 2 Voroshilova str., St. Petersburg, 193318, Russia; OML, Inc., 300 Digital Dr. Morgan Hill, CA 95037, USA. 
[2]. P.H. Siegel "Submillimeter-Wave Camera," JPL New Technology Report, NTR-20718, May 14, 1999

[3]. T.J. Cornwell and P.J. Napier, P.J., "The focal plane coherence function of an imaging antenna and its use in measuring and correcting aberrations," Radio Science vol. 23, no. 5, pp. 739-48, Sept.-Oct. 1988.

[4]. T.J. Cornwell, M.A. Holdaway, J.M. Uson, "Radio-interferometric imaging of very large objects: implications for array design," Astronomy and Astrophysics, vol. 271, no. 2, pp. 697-713, April 1993.

[5]. See several examples in: D. Emerson and T. Payne, editors, "MultiFeed Systems for Radio Telescopes," ASP Conference Series, v. 75, c. 1995.

[6]. U.U. Graf, S. Heyminck, E.A. Michael and S. Stanko, "KOSMA's 490/810 GHz array receiver," 12th Int. Conf. on Space THz Tech., San Diego, CA, pp. 553-562, Feb. 14-16, 2001.

[7]. C. Walker, C. Groppi, A. Hungerford, C. Kulesa, D. Golish, C. Drouet D'Aubigny, K. Jacobs, U. Graf, C. Martin and J. Kooi, "Pole Star: an $810 \mathrm{GHz}$ Array receiver for Astro," 12th Int. Conf. on Space THz Tech., San Diego, CA, pp. 540-552, Feb. 14-16, 2001.

[8]. T. Klein, S.D. Philipp, R. Gusten, F. Gueth, C. Kasemann, D. Lis and T. Phillips, "CHAMP, the carbon heterodyne array of the MPIfR: retrospect and perspectives," SPIE Millimeter and Submillimeter Detectors for Astronomy, v. 4855, 2002.

[9]. S.E. Clark, J.A. Lovberg and J.A. Gallianoo, "Passive millimeter-wave imaging for contra-band detection," SPIE Proceedings, v. 3575, pp. 322-325, Nov. 3-Nov 5, 1998.

[10]. Lee Mirth, A. Pergande, D. Eden and L. Chu, "Passive millimeter wave camera images, current and future," SPIE Proceedings, v. 3703, pp. 6875, April 7, 1999.

[11]. A.H. Lettington, D. Dunn, M. Attia and I. Blankson, "Passive millimeter-wave imaging architectures," J. Opt. A: Pure Appl. Opt., v.5, pp. S103-S110, 2003.

[12]. R. Appleby, "Mechanically scanned real time passive millimeter wave imaging at $94 \mathrm{GHz}$," SPIE vol. 5077, pp. 1-6, 2003.

[13]. L. Yujiri, M. Shoucri, P. Moffa, "Passive millimeter wave imaging," IEEE Microwave Magazine, vol. 4, no. 3, pp. 39-50, Sept. 2003.

[14]. D.B. Rutledge, D.P. Neikirk and D.P. Kasilingam, "Integrated-Circuit Antennas," Chapter 1 of Infrared and Millimeter Waves, vol. 10, Academic Press, pp. 1-90, 1983.

[15]. H.R. Fetterman, T. Sollner, P.T. Parrish, C.D. Parker, R.H. Mathews, P.E. Tannenwald, "Printed Dipole Millimeter Wave Antenna For Imaging Applications," Electromagnetics, vol. 3, pp. 209-215, 1983.

[16]. C.E. Zah, D. Kasilingam, J.S. Smith, D.B. Rutledge, T.C. Wang, S. Schwarz, "Millimeter Wave Monolithic Schottky Diode Imaging Ar- 
rays," International Journal of Infrared and Millimeter Waves, vol. 6, no. 10, pp. 981-997, Oct. 1985.

[17]. G.M. Rebeiz, D.P. Kasilingam, Y. Guo, P. A. Stimson, D. B. Rutledge, "Monolithic Millimeter-Wave Two-Dimensional Horn Imaging Arrays," IEEE Transactions on Antennas and Propagation, vol. 38 no. 9, pp. 1473-1482, Sept. 1990.

[18]. S. Gearhart, C.C. Ling, G.M. Rebeiz, H. Davee, G. Chin, "Integrated 119-um Linear Corner-Cube Array," IEEE Microwave and Guided Wave Letters, vol. 1, no. 7, pp. 155-157, July 1991.

[19]. W.Y. Ali-Ahmad, G.M. Rebeiz, H. Dave, G. Chin, "802 GHz Integrated Horn Antennas Imaging Array," Int. Journal of Infrared \& Millimeter Waves, vol. 12, pp. 481-486, May 1991.

[20]. P.A. Stimson, R.J. Dengler, P.H. Siegel and H.G. LeDuc, "A Planar Quasi-Optical SIS Receiver Suitable for Array Applications," 1992 IEEE MTT-S International Microwave Symposium, Paper MM-4, pp. 1421-24, June 4, 1992.

[21]. C.M. Mann, P. de Maagt, G. McBride, F. van de Water, D. Castiglione, A. McCalden, L. Deias, J. O'Neill, A. Laisne, J.T. Vallinas, I. Ederra, D. Haskett, D. Jenkins, A. Zinn, M. Ferlet and R. Edeson, "Microfabrication of 3D terahertz circuitry," 2003 MTT-S Int. Microwave Sym. Digest, pp. 739-742, June 2003.

[22]. D. T. Petkie, F. C. De Lucia, C. Casto, P. Helminger, E. L. Jacobs, S. K. Moyer, S. Murrill, C. Halford, S. Griffin, and C. Franck, "Active and passive millimeter and sub-millimeter-wave imaging," Proc. SPIE, vol. 5989, pp. 598918-1 to 598918-8, 2005.

[23]. C.M. Mann, "A compact real time passive terahertz imager," SPIE Defense and Security Symposium: Passive Millimeter-Wave Imaging Technology IX, session 4, Orlando, FL, paper 6211-14, April 17-21, 2006.

[24]. W.J. Wilson, R.J. Howard, A. Ibbott, G.S. Parks and W.B. Ricketts, "Millimeter-Wave Imaging Sensor," Trans. on Microwave Theory and Techniques v. 34, no. 10, pp. 1026-1035, Oct. 1986.

[25]. J. W. Waters et.al, "The UARS and EOS Microwave Limb Sounder (MLS) Experiments," Journal of the Atmospheric Sciences, vol. 56, no. 2, pp. 194-218, Feb. 1999.

[26]. Michael C. Gaidis, H.M. Pickett, C.D. Smith, R.P. Smith, S.C. Martin and P.H. Siegel "A 2.5 THz Receiver Front-End for Spaceborne Applications," IEEE Trans. on Microwave Theory and Techniques, vol. 48, no. 4, pp. 733-739, April 2000.

[27]. E.R. Mueller, W.E. Robotham, Jr., R.P. Meisner, R.A. Hart, J. Kennedy and L.A. Newman, "2.5 THz laser local oscillator for the EOS Chem I satellite," Proc. 9th. Int. Conf. on Space THz Tech, p.563, Pasadena, CA, March 1998. 
[28]. The SIFIR-50 is currently available from Coherent Inc., 5100 Patrick Henry Drive, Santa Clara, CA 95054 USA.

[29]. P.H. Siegel and R.J. Dengler, "Terahertz Heterodyne Imager for Biomedical Applications," SPIE Conf. on THz and GHz Electronics and Photonics III, vol. 5354, pp. 1-9, San Jose, CA, Jan 25-26, 2004.

[30]. P.H. Siegel and R.J. Dengler, "Applications \& Early Results from THz Heterodyne Imaging at $119 \mu \mathrm{m}$," Joint 29th Int. Conf. on Infrared and Millimeter Waves, Karlsruhe, Germany, paper 164, Sept. 27 - Oct. 1, 2004.

[31]. A.J. Gatesman, R.H. Giles, and J. Waldman, "A High Precision Reflectometer for the Study of Optical Properties of Materials in the Submillimeter", Proc. of the 17th Int. Conf. on Infrared and Millimeter Waves, Los Angeles, CA, Dec. 1992.

[32]. P.H. Siegel, R.P. Smith, S. Martin and M. Gaidis, "2.5 THz GaAs Monolithic Membrane-Diode Mixer", IEEE Transactions Microwave Theory and Techniques, vol. 47, no. 5, pp. 596-604, May 1999.

[33]. H.M. Pickett, J.C. Hardy and J. Farhoomand, "Characterization of a Dual Mode Horn for Submillimeter Wavelengths," IEEE Trans. Microwave Theory and Techniques, vol. MTT-32, no. 8, pp. 936-8, Aug. 1984.

[34]. J. L. Doane, "Broadband superheterodyne tracking circuits for millimeter wave measurements," Rev. Sci. Instr., vol. 51, no. 3, pp. 317-20, March 1980.

[35]. N.G. Douglas, Millimetre and Submillimetre Wavelength Lasers, A Handbook of CW Measurements, Springer Verlag Series in Optical Sciences v. 61, Berlin, 1989, especially pages 28-32.

[36]. W.R. McGrath, B.S. Karasik, A. Skalare, B. Bumble and H.G. LeDuc, "Hot electron superconductive mixers for THz frequencies," SPIE Terahertz Spectroscopy and Applications conference, vol. 3617, pp. 80-88, Jan. 1999.

[37]. A. Karpov, D. Miller, F. Rice, J. Zmuidzinas, J. Stern, B. Bumble and H.G. LeDuc, "Low noise 1.2 THz SIS receiver," 8th Int. Superconducting Electronics Conference, Osaka, Japan, pp. 521-522, June 19-22, 2001.

[38]. R. Kohler, A. Tredicucci, F. Beltram, H. Beere, E. Linfield, A. Giles Davies, D. Ritchie, A. Lotti and F. Rossi, "Terahertz semiconductorheterostructure laser," Nature, vol. 417, pp. 156-9, May 9, 2002.

[39]. J.R. Gao, J.N. Hovenier, Z.Q. Yang, "Terahertz heterodyne receiver based on a quantum cascade laser and a superconducting bolometer," Applied Physics Letters, vol. 86, no. 24, pp. 244104-1 to 3, 13 June 2005.

[40]. R. A. Witte, Spectrum and Network Measurements, First Noble Publishing, pp. 236-283, 2001. 
[41]. A. Barkan, F. K. Tittel, D. M. Mittleman, R. Dengler, P. H. Siegel, G. Scalari, L. Ajili, J. Faist, H. E. Beere, E. H. Linfield, A. G. Davies, D. A. Ritchie, "Linewidth and tuning characteristics of terahertz quantum cascade lasers," Optics Letters, v. 29, no. 6, pp. 575-577, March 2004.

[42]. Imran Mehdi, P.H. Siegel, D. A. Humphrey, T. H. Lee, R. J. Dengler, J. E. Oswald, A. Pease, R. Lin, H. Eisele, R. Zimmermann and N. Erickson, "An All Solid-State $640 \mathrm{GHz}$ Subharmonic Mixer," IEEE MTT-S Int. Microwave Symposium, Baltimore, MD, pp. 403-406, June 1998.

[43]. P.H. Siegel and I. Mehdi, "The Spare-Diode-Detector: A New PlanarDiode Detector Circuit with State-of-the-Art Performance for Millimeter and Submillimeter Wavelengths," JPL New Technology Report, NPO-20104, Dec. 27, 1996.

[44]. L. Samoska, T. Gaier, A. Peralta, S. Weinreb, J. Bruston, I. Mehdi, Y.C. Chen, H.H. Liao, M. Nishimoto, R. Lai, H. Wang, Y.C. Leong, "MMIC Power Amplifiers as Local Oscillator Drivers for FIRST," Proc. SPIE, vol. 4013, pp. 275-284, 2000.

[45]. H. Wang, L. Samoska, T. Gaier, A. Peralta, H.-H. Liao, Y.C. Leong, S. Weinreb, Y.C. Chen, M. Nishimoto, R. Lai, "Power-Amplifier Modules Covering 70-113 GHz Using MMICs," IEEE Trans. Microwave Theory and Tech., vol. 49, pp. 9-16, 2001.

[46]. N. Wyborn, "The HIFI Heterodyne Instrument for FIRST: Capabilities and Performance," ESA Symposium: The Far InfraRed and Submillimetre Universe, Grenoble, France, pp. 19-24, 15-17 April 1997.

[47]. A. Maestrini, J. Ward, J. Gill, H. Javadi, E. Schlecht, C. TriponCanseliet, G. Chattopadhyay, I. Mehdi, "A 540-640-GHz HighEfficiency Four-Anode Frequency Tripler," IEEE Trans. Microwave Theory and Tech., vol. 53, pp. 2835-2843, 2005.

[48]. Imran Mehdi, P.H. Siegel, D. A. Humphrey, T. H. Lee, R. J. Dengler, J. E. Oswald, A. Pease, R. Lin, H. Eisele, R. Zimmermann and N. Erickson, "An All Solid-State 640 GHz Subharmonic Mixer," IEEE MTT-S Int. Microwave Symposium, Baltimore, MD, pp. 403-406, June 1998.

[49]. P.H. Siegel and I. Mehdi, "The Spare-Diode-Detector: A New PlanarDiode Detector Circuit with State-of-the-Art Performance for Millimeter and Submillimeter Wavelengths," JPL New Technology Report, NPO-20104, Dec. 27, 1996.

[50]. L. Samoska, T. Gaier, A. Peralta, S. Weinreb, J. Bruston, I. Mehdi, Y.C. Chen, H.H. Liao, M. Nishimoto, R. Lai, H. Wang, Y.C. Leong, "MMIC Power Amplifiers as Local Oscillator Drivers for FIRST," Proc. SPIE, vol. 4013, pp. 275-284, 2000.

[51]. H. Wang, L. Samoska, T. Gaier, A. Peralta, H.-H. Liao, Y.C. Leong, S. Weinreb, Y.C. Chen, M. Nishimoto, R. Lai, "Power-Amplifier Modules Covering 70-113 GHz Using MMICs," IEEE Trans. Microwave Theory and Tech., vol. 49, pp. 9-16, 2001. 
[52]. N. Wyborn, "The HIFI Heterodyne Instrument for FIRST: Capabilities and Performance," ESA Symposium: The Far InfraRed and Submillimetre Universe, Grenoble, France, pp. 19-24, 15-17 April 1997.

[53]. A. Maestrini, J. Ward, J. Gill, H. Javadi, E. Schlecht, C. TriponCanseliet, G. Chattopadhyay, I. Mehdi, "A 540-640-GHz HighEfficiency Four-Anode Frequency Tripler," IEEE Trans. Microwave Theory and Tech., vol. 53, pp. 2835-2843, 2005.

[54]. J. R. Birch, J. D. Dromey, J. Lesurf, "The Optical Constants of Some Common Low Loss Polymers Between 4 and $40 \mathrm{~cm}^{-1}$," National Physical Laboratory Report DES 69, February 1981.

[55]. J. A. Lovberg, J. A. Galliano, Jr., R. Olsen, "Passive millimeter-wave imaging using a sparse phased-array antenna," Aerosence Conference: Passive Millimeter Wave Technology, SPIE Proceedings v. 3064, paper 3064-07, 21-22 April 1997.

[56]. B. Agarwal, A.E. Schmitz, J.J. Brown, M. Matloubian, M.G. Case, M. Le, M. Lui and M.J.W. Rodwell, "112-GHz, 157-GHz, and 180-GHz InP HEMT traveling-wave amplifiers," IEEE Trans. on Microwave Theory and Techniques, vol. 46, no. 12, pt. 2, pp. 2553-9, Dec. 1998.

[57]. See for example Passive Millimeter Wave sessions at past SPIE AeroSense Conferences beginning in 1997 with v. 3064, "Passive Millimeter-Wave Imaging Technology" and then starting in 2004, the annual "SPIE Defense and Security Symposium."

[58]. R.J. Dengler, A. Skalare and P.H. Siegel, "Passive and active imaging of humans for contra-band detection at $640 \mathrm{GHz}$," IEEE 2004 IMS Symposium, Fort Worth, TX, June 7-11, 2004.

[59]. P.R. Coward and R. Appleby, "Development of an illumination chamber for indoor millimeter-wave imaging," Proc. SPIE Passive Millimeter-Wave Imaging Technology VI, Orlando, FL, v. 5077, pp. 54-61, 2003. 